\title{
RESPONSE OF COYOTES TO AVIAN DISTRESS CALLS: TESTING THE STARTLE-PREDATOR AND PREDATOR-ATTRACTION HYPOTHESES
}

\author{
by
}

\begin{abstract}
KRISTI K. WISE ${ }^{1)}$, MICHAEL R. CONOVER and FREDERICK F. KNOWLTON ${ }^{2)}$
(Department of Fisheries and Wildlife, Utah State University, Logan, Utah 84322-5210, USA)
\end{abstract}

(Acc. 11-V-1999)

\section{Summary}

We tested two hypotheses proposed to explain why many birds emit distress calls when in the grasp of a predator: the startle-predator and predator-attractionhypotheses. Responses of captive coyotes to a starling distress call were compared between no-call and call trials to determine whether coyotes are startled by the call, and if so, whether they habituated to it. The coyotes were then paired and re-tested to determine whether the call incites a second coyote to approach and interfere with the attack of the initial coyote.

Most coyotes exhibited a startle response during their first exposure to the distress call, their total startle response and total attack time significantly increased, and number of tugs on the prey significantly decreased in initial response to the call. However, distress calls may only startle naive or inexperienced predators because the coyotes habituated rapidly to the distress call playback.

Although coyotes were attracted by the starling distress call, this study provided no evidence indicating that the call caused attracted coyotes to disrupt the attack of the first coyote. Furthermore, when an attracted coyote physically interfered, it frequently induced an intensified attack on the prey by the first coyote.

\section{Introduction}

When captured by a predator, animals may employ a variety of tactics to increase the probability of escape or the survival of their kin. One such tactic

1) Address for correspondence: Kristi Wise, Burns and McDonnell, 9400 Ward Parkway, Kansas City, MO 64114, USA; e-mail address: kwise@burnsmcd.com

2) Thanks to C. Shaw for his assistance collecting data, to J. Gessaman for helpful editing suggestions, and to D. Sisson for statistical support. 
is to produce distress calls. Though many species make distress calls (Bogert, 1960; Hoff \& Moss, 1974; Fenton et al., 1976; Smith, 1977; Staton, 1978; Hauser, 1986), not all species, nor all individuals of a particular species, do so (Norris \& Stamm, 1965). The incidence of distress calls within a species can vary by age, sex, and season (Stefanski \& Falls, 1972a; Balph, 1977).

Among avian species, distress calls are notably similar in structure (Hogstedt, 1983). Most consist of short, repeated bursts of sound covering a wide range of frequencies: characteristics which increase the effective distance (Marten \& Marler, 1977) and locatability of the call (Knudsen, 1980). Each bout generally lasts 5-10 s (Stefanski \& Falls, 1972b; Conover, 1994), but may be repeated. Some species, such as the starling (Sturnus vulgaris), produce relatively continuous distress calls (Perrone, 1980; Conover, 1994).

Four hypotheses have been proposed to explain the function of distress calls:

(1) to request aid from either kin or unrelated reciprocators (request-aid; call-for-help hypothesis) (Rowher et al., 1976);

(2) to warn kin of the nature and location of the predator, thereby enhancing the caller's inclusive fitness by increasing the fitness of its relatives (warn-kin hypothesis) (Rowher et al., 1976);

(3) to startle the predator into releasing the caller (startle-predator hypothesis) (Driver \& Humphries, 1969); and

(4) to attract other predators which distract the attacking predator, enabling the caller to escape (predator-attraction hypothesis) (Curio, 1976; Hogstedt, 1983).

These hypotheses are not mutually exclusive; evidence supporting one hypothesis does not necessarily disprove another. Given the diversity of responses to distress calls recorded for various avian predators, it seems likely there may be multiple functions for distress calling. For instance, it would require little or no additional energy and it could conceivably enhance the opportunity for escape if birds produced distress calls to request aid and startle the predator simultaneously.

Considerable evidence has accumulated supporting or countering the request-aid and warn-kin hypotheses (Stefanski \& Falls, 1972a,b; Rowher et al., 1976; Perrone, 1980; Greig-Smith, 1982; Inglis et al., 1982; Hogstedt, 1983; Hill, 1986; Koenig et al., 1991; Conover, 1994), but they will be discussed only as they may relate to the startle-predator and predatorattraction hypotheses, the primary focus of this study. 


\section{Startle-predator hypothesis}

Aside from Conover's (1994) study which reported that 3 of 15 predators he observed were startled when they first heard a distress call, there are no reports indicating the call startles predators into releasing their live prey. Most support for this hypothesis comes from common-sense predictions that predators should be startled or confused by such an abrupt, loud, unexpected, and ventriloquial noise (Perrins, 1968; Driver \& Humphries, 1969). Conover also provided evidence ( 2 of the 15 predators) that the call provokes an increased attack effort by predators, suggesting the caller has about an equal chance (3vs 2) of startling the predator and escaping, or inspiring a more intense attack and increasing its probability of dying. That a distress call may signal to a predator that its prey is still alive and provoke an intensified attack, rather than frightening it, was first suggested by Perrone (1980).

The startle-predator hypothesis requires that distress calls be abrupt, brief, and explosive. Perrone (1980), Hogstedt (1983), and Conover (1994) reported that these characteristics are evident among the distress calls of most, but not all, species. Continuous calls produced by some species seem to suggest that if this hypothesis is valid, it may not be all-encompassing.

Equally important is that the initiation of the call be unexpected. If predators are indeed startled by distress calls, their responsiveness may diminish over time, with the magnitude of decline dependent upon the rate of exposure. Such habituation would be undesirable to the caller. Likewise, naive predators - those that have rarely or never heard a distress call should be most likely startled by a distress call. The potential effects of novelty and habituation have not been assessed.

\section{Predator-attraction hypothesis}

The predator-attraction hypothesis has garnered support primarily from observations that occasionally predators are attracted to recorded distress calls (Perrone, 1980; Hogstedt, 1983; Koenig et al., 1991). Decoying coyotes, foxes, and other predators with mimicked distress calls is an effective tool for luring predators to hunters, photographers, and field researchers (Alcorn, 1946; Morse \& Balser, 1961). Before choosing to approach a caller in distress, predators presumably must learn to associate the call with easily obtained food (Hogstedt, 1983). Hogstedt observed that 8 young, presumably 
naive, buzzards (Buteo buteo) did not respond to broadcast distress calls, whereas 14 of 24 unaged buzzards approached.

If the predator-attraction hypothesis is valid, some attracted predators should actually interfere with the attacker, and the caller should be able to escape without serious injury. Currently there is only anecdotal evidence that such interference may occur (Koenig et al., 1991).

The primary objectives of this study were to determine whether (1) coyotes (Canis latrans) are startled by the starling distress call; (2) coyotes become habituated by repeated exposures to distress calls; (3) a secondary coyote is attracted to a distress call, and if so, whether the attracted coyote influences the behavior of the coyote first to attack (primary coyote); and (4) sexual differences in coyote responses to a distress call are evident.

\section{Methods}

The startle-predator hypothesis was investigated by examining the responses of individual coyotes during trials with and without a starling distress call. Investigation of the predatorattraction hypothesis was identical to that for the startle-predator hypothesis, except coyotes were paired to investigate their responses to both the distress call and their mates.

Test subjects included 38 (20 males, 18 females) captive, hand-raised, adult coyotes approximately 1.5 years of age. Coyotes were maintained in male-female pairs throughout the study except as dictated for individual trials. We have no specific knowledge that any of the coyotes had ever either attacked prey or were exposed to distress calls.

Coyotes were chosen as test subjects because they commonly associate either in pairs or packs (Bekoff \& Wells, 1986) and generally remain in close proximity when hunting, potentially enhancing the predator interactions necessary to investigate the predator-attraction hypothesis. Coyotes are also generalist predators, occasionally attacking and eating starlings and other similar birds when the opportunity arises. We purposely selected a generalist because such a predator would likely be more inexperienced and naive to distress calls than typical passerine predators and thus might be most affected by the call.

Coyotes and facilities utilized during this study were provided by the U.S. Department of Agriculture's Predator Research Facility located $10 \mathrm{~km}$ south of Logan, Utah. This study was conducted in 18 0.1-ha teardrop-shaped pens arranged in groups of 3 around a central observation building.

Carcasses of 3-week-old chickens, approximately the size of adult starlings, were presented to the coyotes during each trial from atop a 3-m post. Use of carcasses, rather than live birds, facilitated compliance with animal care and use procedures. Chicken poults were fastened to a metal ring using a double strand of $22 \mathrm{~kg}$-test monofilament wrapped around the poult's midsection. Immediately prior to each trial, the ring was slipped over the post and secured at the top with a hook. Trials were initiated by pulling on monofilament line extending to the hook from an observation vehicle, thereby releasing the ring from the hook and allowing the bird and ring to slide down the post to the ground. 
The distress call was broadcast during call trials by a speaker placed under a plastic crate secured at the base of the post. The recording of the distress call (R.S. Little, (C) 1994 All rights reserved. Library of Natural Sounds, Cornell Laboratory of Ornithology, Ithaca, New York) consisted of a virtually-continuous distress call produced by one or occasionally several starlings. The intensity of the call was between 90 and $95 \mathrm{~dB}$ when measured with a soundlevel meter $1.5 \mathrm{~m}$ from the speaker, the distance at which coyotes attacked the bird. The distress call was initiated the moment the coyote made physical contact with the bird carcass and was broadcast for approximately $5 \mathrm{~s}$.

Each trial was recorded with a video camera mounted on a tripod outside the pen to allow evaluation of behaviors which were difficult to record during the trial.

\section{Individual trial protocol}

All coyotes were tested individually to investigate the startle-predator hypothesis between 29 September and 21 November 1994. Prior to initiation of the experimental trials, 2-6 days of conditioning trials without distress calls were conducted to habituate the animals to the apparatus and presentation scenario.

The experimental regime for each coyote consisted of a sequence of 8 trials conducted on consecutive days. Treatment I (no-call treatment), involving 4 trials with no distress call playback, was immediately followed by treatment II (call treatment), composed of 4 trials during which the distress call was played when the coyote made physical contact with the chicken carcass. No-call and call trials were not randomized so habituation to the presentation scenario and distress call could be investigated.

The order in which trials among coyotes were conducted on any specific day were systematically randomized to ensure all coyotes were tested during different times of the morning. Each day's trials were completed before the coyotes were fed their standard daily ration to avoid potential complications associated with satiation.

\section{Response variables}

Each coyote's behavior was recorded from the moment the bird landed on the ground until the entire bird was removed from the post. Because we could not directly determine when coyotes killed their 'prey' (the birds were not alive), we assumed the probability a bird might escape was related to hesitation by the coyotes, the vigor of the coyotes' attacks, and the frequency of startle responses exhibited by coyotes to the distress call.

Variables indicative of a coyote's hesitation and/or eagerness to attack during trials included attack initiation time, frequency of attacks and tugs on the bird, total attack time, and subjective estimates of bite intensity and the bird's survival time, which was defined as the elapsed time from the coyote's first attack until the attack after which the 'prey' likely could not have survived (i.e. when the coyote removed a substantial portion of the carcass, such as a leg or wing). Various degrees of a startle response were indicated by back-aways, dropbirds, flinches, jump-backs, and pause-attacks and were additively combined into a single 'total-startle-response' category for analysis of the overall startle response during no-call and call trials. In addition, the intensity of the coyotes' startle response to the distress call during trials 5-8 was subjectively ranked on a 0 (no response) to 10 (extremely startled) scale (startle rank). 


\section{Statistical analyses}

Statistical comparisons between no-call and call treatments, between all trials (1-8) regardless of treatment, and between gender were made using a split-plot unbalanced design (due to missing data) ANOVA, using the SAS GLM procedure (SAS Institute, 1985). These analyses required class variables of gender, coyote, treatment, and trial; trial was nested within treatment and coyote was nested within gender. All ANOVAs were applied to logtransformed data, and an alpha value of 0.05 was used to determine significance except when inappropriate, as noted below.

Trial 4 (final no-call trial) and trial 5 (first call trial) were contrasted to identify the immediate response of coyotes to the distress call while avoiding complications arising from possible habituation during later trials. To identify whether adult coyotes habituated to the distress call during call trials, data from trials 5 and 8 of the call treatment were contrasted; trials 1 and 4 were contrasted to determine whether habituation to the apparatus and scenario was evident. Because 3 contrasts were investigated, it was appropriate to use a Bonferroniprotected alpha value of 0.016 , obtained by dividing 0.05 by the number of contrasts, for determining significance between trials.

\section{Pair trial protocol}

Immediately after completing the final call trial (trial 8) of the individual coyote experiment, the distress call was played when each coyote was some distance from the apparatus to determine whether the coyotes associated the call with the chicken poult and were attracted by it. No bird was presented to the coyotes at this time.

Following completion of individual trials, all subjects were paired with their mates in preparation for trials to investigate the predator-attraction hypothesis, completed between 30 November and 15 December 1994. Like the individual trial protocol, this experiment consisted of 4 trials in which no distress call was played followed by 4 trials during which the distress call was broadcast.

\section{Response variables}

Coyote responses were recorded from just before the release of the bird from the post until the birds' removal by a coyote. Attacks, attack initiation time, and total attack time were recorded identically to the individual coyote experiment.

The degree of intolerance of the primary coyote to its mate during pair trials was measured by mate approaches, mate attacks, chases, threats, and pauses in the coyotes' attack on the bird. These were additively combined into a single 'total attack interruptions' category for analysis of the overall influence of the secondary coyote on the primary coyote's attack on the bird. In addition, a value between 0 (no interaction observed) and 10 (attacks and chases observed frequently) was subjectively given each pair corresponding to the frequency and intensity of their interactions. Approaches by the secondary coyote within $1.5 \mathrm{~m}$ of the primary coyote were recorded as an indicator of the secondary coyotes' interest in the bird, the distress call, and the primary coyotes' activities.

\section{Statistical analyses}

Differences between treatments, trials, and gender were identified using a split-plot unbalanced design ANOVA on log-transformed data using gender, pair, treatment, and trial as class variables; trial was nested within treatment. 
A split-plot unbalanced design ANOVA with class variables treatment, gender, test (individual experiment $v s$ pair experiment), and coyote nested within a gender by treatment interaction was used to identify differences between attack characteristics of those coyotes attacking the bird during the pair trials and their attack characteristics when alone (during individual trials).

\section{Results}

Startle-predator trials

Seventeen of 30 coyotes $(57 \%)$ were clearly startled (exhibited one or more of the startle response variables) by the distress call during their first exposure (trial 5) in the individual coyote experiment, but this response decreased during successive trials. Seven (23\%) did not visibly respond when the distress call was played during trial 5, and 6 (20\%) responded only weakly (flinched, but infrequently). One coyote did not make another attack on the bird throughout the remaining 3 trials of the call treatment following her initial and only attack in trial 5.

The survival time of the bird was significantly longer and the number of coyotes' attacks declined during the trials when the distress call was played versus the no-call trials (Table 1), suggesting that the distress call may increase a bird's likelihood of escape. In contrast, the total startle response (TSR), attack initiation time, and number of tugs exhibited by the coyotes were not significantly different between the no-call and call treatments when all trials were included (Table 1).

When trials 4 and 5 (or trials 5 and 6 for attack initiation time) were contrasted individually, survival time, attack initiation time, bite intensity and number of attacks did not change significantly, but the TSR and total attack time significantly increased and number of tugs decreased (Table 2). Trials 5 and 6, rather than trials 4 and 5, were contrasted for attack initiation time because measurement of this variable preceded the playback of the distress call: the coyotes had not previously heard the call during their approach in trial 5 .

Contrasts between trials 1 and 4 were not significant for any variable (Table 2), suggesting the coyotes did not alter their behavior in response to the apparatus during this treatment. On the other hand, differences between trials 5 and 8 were significant, or nearly so $(p<0.06)$, for all variables (Table 2). The TSR declined significantly (largely between trials 5 and 
TABLE 1. Means $(S E)^{a}$ for variables measured during no-call and call treatments during the individual coyote trials

\begin{tabular}{|c|c|c|c|c|}
\hline \multirow[b]{2}{*}{ Response variable } & \multicolumn{2}{|c|}{ Treatment $(N=120)$} & \multirow[b]{2}{*}{$F$} & \multirow[b]{2}{*}{$p$} \\
\hline & No-call & Call & & \\
\hline Survival time (s) & $2.18 \quad(0.44)$ & $3.76(0.65)$ & 4.35 & 0.04 \\
\hline Total startle response & $2.60 \quad(0.21)$ & $3.05 \quad(0.24)$ & 1.99 & 0.16 \\
\hline Initiation time (s) & $31.77 \quad(2.64)$ & $33.05 \quad(2.82)$ & 0.11 & 0.74 \\
\hline Bite intensity ${ }^{\mathrm{b}}$ (rank) & $5.07 \quad(0.16)$ & $5.05(0.16)$ & 0.01 & 0.92 \\
\hline Total attack time $(\mathrm{s})^{\mathrm{b}}$ & $171.67(10.75)$ & $187.82(11.70)$ & 0.91 & 0.34 \\
\hline Attacks (no.) & $9.94 \quad(0.49)$ & $7.76(0.39)$ & 12.78 & 0.0004 \\
\hline Tug bird (no.) & $1.02(0.09)$ & $1.06(0.09)$ & 0.12 & 0.73 \\
\hline
\end{tabular}

${ }^{a}$ Means and standard errors in original units are approximate because antilogarithmic transformation of previously log-transformed values results in biased estimates of $\mu$ and asymmetrical standard errors. These values assume symmetrical standard errors.

$\mathrm{b}$ There was a significant treatment by gender interaction for bite intensity and total attack time.

6), bite intensity and tugs increased significantly, and the rank of coyotes' overall startle response significantly decreased between trials 5 and 8 ( $\alpha=$ 0.02). Similar, though insignificant, patterns were apparent for most other variables.

There were no significant differences between males and females during individual trials except for 2 occasions when a significant interaction between gender and treatment occurred for bite intensity and total attack time (bite intensity: $F=9.71, p=0.002$, df $=1,194$; total attack time: $F=5.09$, $p=0.03, \mathrm{df}=1,196)$. During no-call treatment trials, males (bite intensity: $\bar{x}=4.85, \mathrm{SE}=0.20$; total attack time: $\bar{x}=158.18, \mathrm{SE}=13.53, N=64)$ and females (bite intensity: $\bar{x}=5.30, \mathrm{SE}=0.23$; total attack time: $\bar{x}=187.03$, $\mathrm{SE}=17.08, N=56$ ) did not differ statistically in either bite intensity ( $p=$ $0.15)$ or total attack time $(p=0.18)$. During the call treatment trials males were more intense in their attacks (bite intensity: $\bar{x}=5.52, \mathrm{SE}=0.23, p=$ 0.004; total attack time: $\bar{x}=140.92, \mathrm{SE}=12.06, p=0.0001)$ than were females (bite intensity: $\bar{x}=4.62, \mathrm{SE}=0.21$; total attack time: $\bar{x}=248.54$, $\mathrm{SE}=22.67)$. Both males $(p=0.03)$ and females $(p=0.03)$ significantly altered their bite intensities between the no-call and call treatments, though males' bites became more intense and females' less intense. The total attack time of females significantly $(p=0.03$ ) increased across treatment, while that of males did not change $(p=0.34)$. 


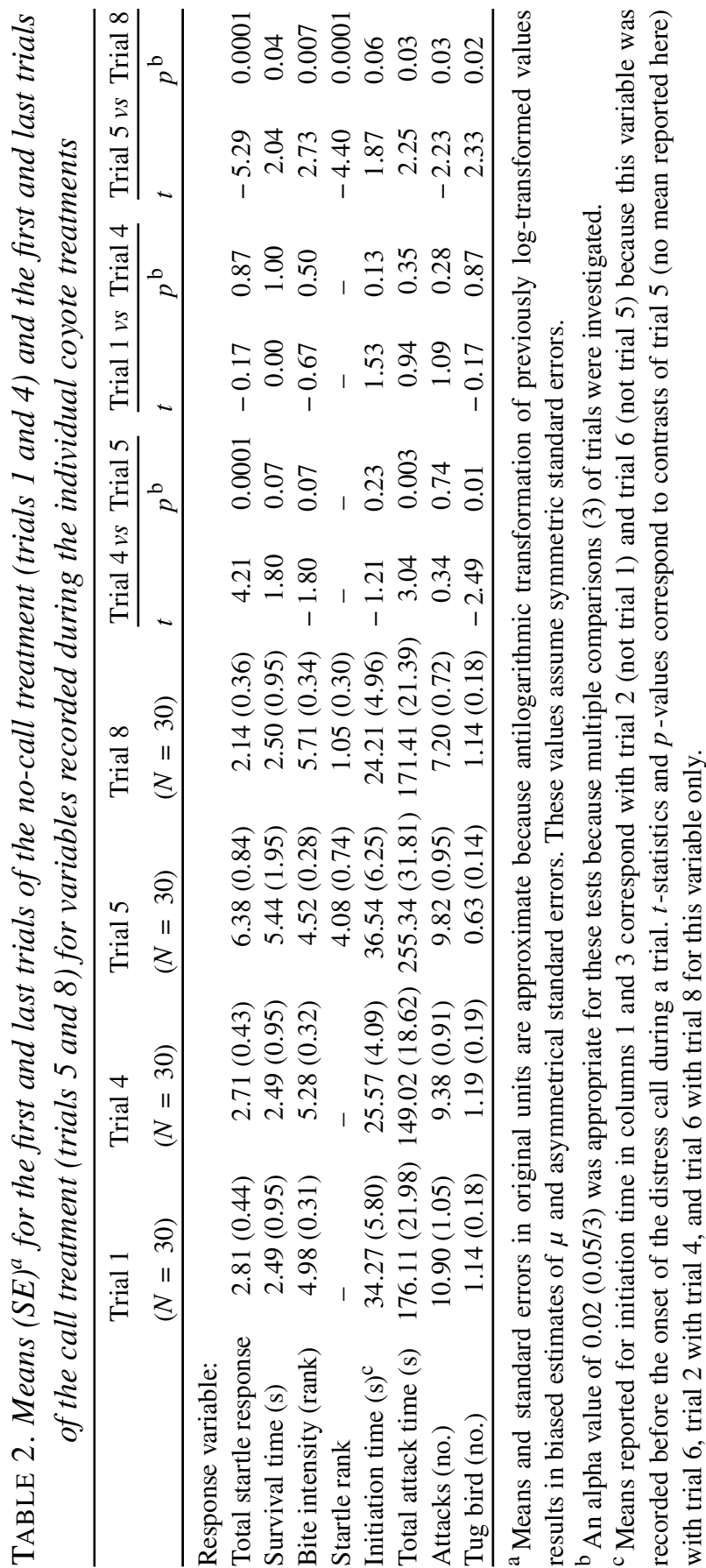




\section{Predator-attraction trials}

Before a coyote can interfere with the attack of another and possibly usurp the bird, it must first be attracted to the distress call. Following the last call trial during the individual coyote experiment, 22 of $26(85 \%)$ coyotes immediately returned (within $15 \mathrm{~s}$ ) to the post after hearing the distress call. Only 2 of these coyotes (8\%) approached the post during a 15-s control interval immediately preceding the distress call playback. During the pairedcoyote experiment, however, secondary coyotes did not approach to within $1.5 \mathrm{~m}$ of the primary coyote (and the bird) more frequently when the distress call was broadcast than they did when no call was played $(F=1.79, p=$ $0.18)$.

Furthermore, none of the variables representing interruptions in the primary coyote's attack on the bird (total attack interruptions, mate chases, attacks, threats, mate approaches, and pauses in bird attacks) were significantly different between call and no-call treatments (Table 3).

The same member of each pair generally attacked the bird throughout all trials. Seventeen coyotes among the 17 pairs made $95 \%$ of all attacks on the bird. Most (76\%) primary coyotes were also males $(z=6.15, p=0.0001)$. The female in only one pair consistently initiated the attack on the bird. Not surprisingly, given this gender bias, females differed from males in most of

TABLE 3. Means $(S E)^{a}$ for attack interruption variables during no-call and call treatments for paired coyotes

\begin{tabular}{llll}
\hline & Treatment $(N=136)$ & & \\
\cline { 2 - 3 } & No-call & Call & \\
\hline Response variable: & & & \\
Total attack interruptions & $0.65(0.08)$ & $0.52(0.07)$ & 0.19 \\
$\quad$ Chase mate & $0.10(0.03)$ & $0.08(0.03)$ & 0.46 \\
Attack mate & $0.07(0.02)$ & $0.05(0.02)$ & 0.37 \\
Approach mate & $0.13(0.03)$ & $0.12(0.03)$ & 0.61 \\
Threaten mate & $0.12(0.03)$ & $0.11(0.03)$ & 0.96 \\
$\quad$ Pause attack & $0.21(0.03)$ & $0.18(0.03)$ & 0.17 \\
Rank of interactions & $4.39(0.61)$ & $3.82(0.55)$ & 0.49 \\
\hline
\end{tabular}

${ }^{a}$ Means and standard errors in original units are approximate because antilogarithmic transformation of previously log-transformed values results in biased estimates of $\mu$ and asymmetrical standard errors. These values assume symmetrical standard errors. 
their responses, including the number of attacks $(F=19.81, p=0.0004$, $\mathrm{df}=1,16)$ and total attack interruptions $(F=19.19, p=0.0005)$.

\section{Individual versus pair tests}

To determine if the presence of a coyote's mate altered the primary coyote's attack behavior, responses recorded during both individual and pair experiments were compared. Number of attacks and total attack times were compared among coyotes (both primary and secondary) which attacked the bird, and attack initiation times were compared only among primary coyotes.

The difference between individual and pair tests for total attack times was gender-dependent $(F=28.19, p=0.0001, \mathrm{df}=1,183)$ : the attack times of both genders greatly decreased from the individual (males: $\bar{x}=143.99$, SE $=13.33$; females: $\bar{x}=180.49, \mathrm{SE}=40.12$ ) to pair experiment (males: $\bar{x}=$ 50.35, SE $=4.72$; females: $\bar{x}=10.31, \mathrm{SE}=2.50$ ).

Similarly, the number of attacks made by attacking males and females significantly decreased $(F=51.43, p=0.0001, \mathrm{df}=1,183)$ from individual $(\bar{x}=9.36, \mathrm{SE}=0.70)$ to pair tests $(\bar{x}=4.42, \mathrm{SE}=0.36)$, and the attack initiation time of primary attackers was significantly faster $(F=194.53$, $p=0.0001, \mathrm{df}=1,202)$ during the paired coyote experiment $(\bar{x}=2.30$, $\mathrm{SE}=0.36)$ than during the individual coyote experiment $(\bar{x}=22.57, \mathrm{SE}=$ 2.51).

\section{Discussion}

\section{Startle-predator hypothesis}

The highly significant increase in total startle response and attack times, and the decline in tugs between trial 4 and trial 5 in immediate response to the distress call indicate the coyotes were adversely affected by the distress call. In addition, the majority of coyotes were initially startled during their first exposure to the distress call, though their responses differed in degree.

No significant difference in survival time between trials 4 and 5 suggests that the coyotes did not initially alter the speed at which they 'killed' their prey despite being startled by the distress call. However, when pooled over all call trials the survival time was significantly longer than during the nocall trials, apparently signifying a reduction in the coyotes' attack intensities in response to the distress call over time. 
The startle responses recorded during this study augment those of Conover (1994), except that no immediate increase in attack intensity was observed for captive coyotes in this study, perhaps because they were hand-raised in captivity and naive to distress calls. Conover's raccoons were wild-caught and tested within 2 months of capture, and may have been exposed to distress calls prior to capture.

Males seemed somewhat less affected by the call than females. The bite intensity of females decreased and their total attack times increased during the call treatment, responses coinciding with predictions of the startlepredator hypothesis. In contrast, males did not alter their attack times and increased their bite intensities in response to the call.

The result of combining all trials of a particular treatment for comparison (Table 2) frequently differed from that of comparisons between trials 4 and 5 alone, suggesting a change in coyote behavior between trials 5 and 8. Declines in total startle response and bite intensity, and the increase in tugs through the succession of call trials likely indicated that habituation occurred. Furthermore, the greatest change in most recorded variables generally occurred between trials 5 and 6 , suggesting that habituation to the distress call occurred quickly (i.e. by the coyotes' second exposure to the call). The speed at which habituation to the distress call occurs may differ depending on the rate of exposure.

The response of free-ranging coyotes may somewhat differ from that observed for captive coyotes because they may be more likely to learn that novel objects or occurrences are sometimes hazardous, and a distress call will likely be accompanied by unexpected or sudden movements by the bird in the wild. In addition, the captive coyote's responses may have been affected by their naivete in capturing prey as well as the distress call. Coyotes may respond differently to ground-nesting bird or mammalian distress calls which are more common coyote prey.

\section{Predator-attraction hypothesis}

There are 3 conditions which must occur if the predator-attraction hypothesis is valid: predators must be attracted to distress calls; attracted predators must interfere with the attack of the initial predator; and the bird must be able to occasionally escape.

Most of the coyotes in this study returned to the test apparatus when the distress call was played following the final trial of the individual test, 
suggesting they were attracted to the starling distress call after associating it with the bird. When we tested coyotes in pairs, we were unable to release the bird or broadcast the distress call without both coyotes being immediately aware and therefore could not control to which coyote the bird was presented. The male, perhaps the dominant, was most often the primary attacker within a coyote pair; if not, the male often attacked the female between attempts on the bird, whereas females very rarely attacked their mates. The secondary coyote also rarely approached the primary coyote and the bird during the paired coyote experiment, regardless of the distress call. It seems reasonable that large or dominant predators would be less inclined to release prey to a smaller or subordinate predator because of less risk of being injured and losing the bird. A smaller predator might also be more likely to avoid the probable risk of injury in an altercation with a larger predator.

Only Koenig et al. (1991) have reported interference of an attracted predator with a primary predator. They observed a Cooper's hawk (Accipiter cooperii) capture an acorn woodpecker (Melanerpes formicivorus) and release it after hearing its distress call and spotting the human observer. Their observation is anecdotal and may be confounded by differences in a predator's response to such a threatening predator as a human versus other more typical predators.

During this study, a close approach was not always necessary for the primary coyote to be distracted from its attack on the bird. Although some interruptions of a primary coyote's attack were observed, none of the variables recorded were significantly different between treatments, suggesting that such interruptions were not a result of the distress call. The lack of a treatment difference for approaches to within $1.5 \mathrm{~m}$ of primary coyotes by secondary coyotes further confirms the lack of interest in interfering by secondary coyotes.

We do not have a precise measure of how long it takes a coyote to either kill or severely injure starling-sized prey, though we presume it would be relatively quick once the coyote seized such a small bird. The average survival time of the 'prey' recorded during this study ranged from 0 to just over 6 s. Perrone (1980) noted that various raptor species required at least $10 \mathrm{~s}$ to approach a distress call playback. These time estimates pose a problem for both the request-aid and the predator-attraction hypotheses because there is so little time for the intended recipient of the call to arrive before the caller is killed. In our tests, interruptions caused by secondary 
coyotes were not immediate: the primary coyote usually attacked the bird several times before being distracted.

In addition, there was often an increase in the primary coyotes' attack intensities resulting from the secondary coyotes' display of interest, especially when both coyotes attacked the bird simultaneously. Rarely was the bird released in these instances. The coyotes that attacked the bird during the pair trials (mostly males) were also much more aggressive (significantly faster attack initiation and total attack times and significantly fewer attacks) than these same individuals were when attacking individually. This may be a result of the experience gained during the individual trials, but it probably also includes a competitive component associated with the other coyote. Consequently, a bird probably would be killed more quickly when the coyote's mate is nearby and any interruption in the coyote's attack would likely be after the bird was killed.

To summarize, both the startle-predator and predator-attraction hypotheses have merit in only limited situations. Most coyotes were initially startled by the distress call but quickly habituated to it, suggesting that only inexperienced coyotes may be affected. Coyotes were also attracted by the distress call, but this frequently resulted in a more intense attack on the prey.

\section{References}

Alcorn, J.R. (1946). On the decoying of coyotes. - J. Mammal. 27, p. 122-126.

Balph, M.H. (1977). Sex differences in alarm responses of wintering evening grosbeaks. Wilson Bull. 89, p. 325-327.

Bekoff, M. \& Wells, M.C. (1986). Social ecology and behavior of coyotes. - Adv. Study Behav. 16, p. 251-338.

Bogert, C.M. (1960). The influence of sound on the behavior of amphibians and reptiles. In: Animal sounds and communication (W.E. Lanyon \& W.N. Tavolga, eds). Am. Inst. Biol. Sci. Pub. No. 7, Wash., D.C., p. 137-320.

Conover, M.R. (1994). Stimuli eliciting distress calls in adult passerines and response of predators and birds to their broadcast. - Behaviour 131, p. 19-37.

Curio, E. (1976). The ethology of predation. - Springer Verlag, Berlin, $250 \mathrm{p}$.

Driver, P.M. \& Humphries, D.A. (1969). The significance of the high-intensity alarm call in captured passerines. - Ibis 111, p. 243-244.

Fenton, M.B., Belwood, J.J., Fullard, J.H. \& Kunz, T.H. (1976). Responses of Myotis lucifugus (Chiroptera: Vespertilionidae) to calls of conspecifics and to other sounds. - Can. J. Zool. 54, p. 1443-1448.

Greig-Smith, P.W. (1982). Distress calling by woodland birds. - Anim. Behav. 30, p. 299301. 
Hauser, M.D. (1986). Male responsiveness to infant distress calls in free-ranging vervet monkeys. - Behav. Ecol. Sociobiol. 19, p. 65-71.

Hill, G.E. (1986). The function of distress calls given by tufted titmice (Parus bicolor): an experimental approach. - Anim. Behav. 34, p. 590-598.

Hoff, J.G. \& Moss, S.A. (1974). A distress call in the bullfrog, Rana catesbeiana. - Copeia 1974, p. 533-534.

Hogstedt, G. (1983). Adaptation unto death: function of fear screams. - Am. Nat. 121, p. $562-570$.

Inglis, I.R., Fletcher, M.R., Feare, C.J., Greig-Smith, P.W. \& Land, S. (1982). The incidence of distress calling among British birds. - Ibis 124, p. 351-355.

Knudsen, E.I. (1980). Sound localization in birds. — In: Comparative hearing in vertebrates (A.N. Popper \& R.R. Fay, eds). Springer, New York, p. 289-322.

Koenig, W.D., Stanback, M.T., Hooge, P.N. \& Mumme, R.L. (1991). Distress calls in the acorn woodpecker. - Condor 93, p. 637-643.

Marten, K. \& Marler, P. (1977). Sound transmission and its significance for animal communications. I. Temperate habitats. - Behav. Ecol. Sociobiol. 2, p. 271-290.

Morse, M.A. \& Balser, D.S. (1961). Fox calling as a hunting technique. — J. Wildl. Manage. 25, p. $148-154$.

Norris, R.A. \& Stamm, D.D. (1965). Relative incidence of distress calls or 'squeals' in mistnetted birds. - Bird-banding 36, p. 83-88.

Perrins, C.M. (1968). The purpose of high-intensity alarm calls in small passerines. — Ibis 110 , p. 200-201.

Perrone, M. (1980). Factors affecting the incidence of distress calls in passerines. - Wilson Bull. 92, p. 404-408.

Rowher, S., Fretwell, S.D. \& Tuckfield, R.C. (1976). Distress screams as a measure of kinship in birds. - Am. Midl. Nat. 96, p. 418-430.

SAS Institute, Inc. (1985). SAS user's guide: statistics. — Cary, N.C. 956 p.

Smith, A.K. (1977). Attraction of bullfrogs (Amphibia, Anura, Ranidae) to distress calls of immature frogs. - J. Herpetol. 11, p. 234-235.

Staton, M.A. (1978). 'Distress calls' of crocodilians — whom do they benefit? — Am. Nat. 112, p. 327-332.

Stefanski, R.A. \& Falls, J.B. (1972a). A study of distress calls of song, swamp, and whitethroated sparrows (Aves: Fringillidae). I. Intraspecific responses and functions. - Can. J. Zool. 50, p. 1501-1512.

- _ \& - - (1972b). A study of distress calls of song, swamp, and white-throated sparrows (Aves: Fringillidae). II. Interspecific responses and properties used in recognition. Can. J. Zool. 50, p. 1513-1525. 